with global delay with no spasticity the reports were likely to be similar. Subtle white matter abnormalities were also more likely to be detected by the specialist.

\section{G194 VITAMIN D AND VITAMIN D DEFICIENCY: HOW MUCH DO PARENTS KNOW?}

${ }^{1} \mathrm{R}$ Carr, ${ }^{2} \mathrm{D}$ Mahmood, ${ }^{3} \mathrm{~A}$ McEvoy. ' $S c h o o l$ of Medicine, University of Manchester, Manchester, UK; ${ }^{2}$ Paediatric Department, Lancashire Teaching Hospitals Trust, Preston, UK; ${ }^{3}$ Orthopaedic Department, Lancashire Teaching Hospitals Trust, Preston, UK

\subsection{6/archdischild-2015-308599.188}

Background Vitamin D deficiency is prevalent among children and the majority are unaware of their low vitamin D levels. Vitamin D is an important component for the optimal health of children, as well as playing a role in reducing the risk of various chronic health issues in the future. This study aimed to gauge the level of awareness and knowledge of vitamin D amongst the parents of paediatric orthopaedic patients.

Study design A retrospective observational study was conducted using a paper-based questionnaire to assess parental awareness. The questionnaire was distributed to parents of children attending paediatric orthopaedic clinics in a single Teaching Hospital Trust over a period of four months.

Results 220 parents responded to the questionnaire. $85 \%$ of respondents believed vitamin $\mathrm{D}$ to be important for the health of a child. $65 \%$ said they knew what vitamin D was. $40 \%$ of all parents asked were unable to write a brief statement of their basic understanding surrounding vitamin D. $17 \%$ give their children vitamin D supplementation. 2\% of the respondents' children have been told they are vitamin D deficient.

The participants were asked what they believed to be good dietary sources of vitamin D. $64 \%$ of respondents could identify oily fish or eggs. However, $46 \%$ believed dairy products to be a good dietary source of vitamin D.

The questionnaire asked respondents whether GPs have provided education or advice about the importance of maintaining adequate vitamin D levels in children, only $9 \%$ of parents said that they had received information from their child's GP.

$59 \%$ of respondents to the questionnaire wanted more information about vitamin D and vitamin D deficiency. This information was sent to each of those respondents as a short leaflet.

Conclusion Parental awareness of vitamin D and deficiency is poor. There is a need for increased levels of parental education to ensure children have a better chance of maintaining adequate vitamin D levels.

\section{G195 WHAT ARE THE LONG TERM CLINICAL OUTCOMES OF SPINAL DYSRAPHIAS IN CHILDREN?}

S Pal, H Fernandes, AD Sansome. Department of Paediatrics, Cambridge University NHS Foundation Trust, Cambridge, UK

\subsection{6/archdischild-2015-308599.189}

Background Spinal dysraphia is a non-fatal foetal anomaly comprising a range of spinal canal fusion abnormalities. There have been recent advances in the diagnosis, treatment and prevention of this condition. The longer term outcomes currently vary within the literature and are based historic cohorts prior to more recent advances in care.

Aims Review the long term outcomes and symptomatology in a current cohort of children with spinal dysraphia.
Methods Retrospective review of medical records of patients with diagnosis of spinal dysraphias. Children were excluded if there was other genetic abnormality or syndrome.

Results We identified 70 children (mean age 10.7, SD 7.1, 41\% male). There was $100 \%$ survival at 1 year of age and $68(97 \%)$ children were still alive at time of the review. Mortality in both children was secondary to meningitis. Diagnosis was made antenatally in $37 \%$, and postnatally in $63 \%$ with 3 children $(5.7 \%)$ being diagnosed at school age. $51(73 \%)$ were symptomatic and $22(31 \%)$ had evidence of neuropathic bladder and bowel. Antenatal diagnosis $(p=0.047)$, higher level of lesion $(p=0.044)$ or shunts in situ $(\mathrm{p}<0.001)$ were all predictive of greater symptomatology. If a patient was found to be symptomatic in one area, they were significantly more likely to also have other symptoms $(\mathrm{p}<0.001)$ supporting the need for regular review in these children. There was a high incidence of urinary symptoms (11.7\%) constipation (37\%) and orthopaedic problems (such as scoliosis or lower limb abnormalities) (48\%) in the absence of neuropathy. Mental health issues were documented in 4 children (5.7\%).

Conclusions Spinal dysraphia is associated with high rates of morbidity $(73 \%)$ with a significantly lower mortality (3\%) and incidence of mental health issues $(6 \%)$ when compared to previous studies. The current follow-up of patients with spinal dyraphias varies, therefore further studies are needed to develop appropriate follow-up strategies for this high risk patient group; including the evaluation for comorbidities and psychosocial complications.

\section{G196 WHAT DO CHILDREN AND YOUNG PEOPLE WITH LEARNING DISABILITIES WANT FROM HOSPITAL SERVICES?}

${ }^{1} \mathrm{~K}$ Oulton, ${ }^{1} \mathrm{D}$ Sell, ${ }^{1} \mathrm{~S}$ Kerry, ${ }^{1,2} \mathrm{~F}$ Gibson. ${ }^{1}$ Centre for Outcomes and Experience Research (ORCHID), Great Ormond Street Hospital NHS Foundation Trust, London, UK; ${ }^{2}$ Department of Children's Nursing, London South Bank University, London, UK

\subsection{6/archdischild-2015-308599.190}

Background Children and young people (CYP) with learning disability (LD) have more hospital admissions and greater lengths of hospital stay than children without disabilities. Yet, few researchers have sought to understand the hospital experience from the perspective of these patients, which means we have little evidence of how to improve hospital services in order to meet their needs. Limited research has been conducted with parents of CYP with LD to ascertain what it is like for them and their child to recieve hospital services, which largely reveals parental concerns about staff members lack of confidence and knowledge to care for their child with LD.

Aims Our study sought to understand the hospital related needs and experiences of CYP with LD, their families and hospital staff. Reported here is data collected through observation, interaction and communication with CYP.

Methods An ethnographic approach was used to observe, in detail, the everyday life of 9 CYP with LD and their families during one or more episodes of hospitalisation, over a 12 month period. The use of arts based methods facilitated 4 of these CYP to share what was important to them. 135 staff consented to be observed during this time frame. Interviews with 27 staff, not reported here, were also conducted. Data were analysed using the Framework Approach.

Results Data revealed that despite sharing a label of $\mathrm{LD}$, this population of patients are not all the same and can want different things from hospital services. The key to getting it right is ensuring that their non-medical needs are identified, understood 
and prioritised by hospital staff and the "little things" that are extremely important to these patients are not overlooked. Their need for routine, familiarity and sensory and play facilities was evident. Without their care and treatment being individualised to meet their very specific needs, CYP with LD can experience unnecessary emotional distress and physical harm.

\section{G197(P) NEUROIMAGING IN NEUROFIBROMATOSIS TYPE 1: OUTCOMES FROM A TARGETED APPROACH- EXPERIENCE OF A SINGLE TERTIARY CENTRE}

R Mithyantha, H Royden, J Acharya, Z Bassi. Community Paediatrics, Alderhey Children's Hospital NHS Tust, Liverpool, UK

\subsection{6/archdischild-2015-308599.191}

Aims Routine imaging is not recommended for patients with neurofibromatosis 1 (NF1) and a clinical approach is used to guide need for investigations. We aimed to determine clinical characteristics and symptoms which were most commonly associated with abnormalities on neuroimaging in children with NF1.

Methods We analysed 100 consecutive patients with an established diagnosis of NF1 seen in a tertiary NF1 clinic. Records of patients who had undergone imaging studies were reviewed to determine clinical presentation and associated comorbidities, along with neuroimaging results.

Results 59/100 children with NF1, (M:F = 55:45; median age 11.6 years (range 9 months-21 years) underwent cranial MRI scanning. The most common indications for MRI request being visual disturbance or abnormal fundoscopy (37\%), concerns regarding growth or puberty $(13.5 \%)$ and headaches $(10 \%)$. $91 \%$ had an abnormality on MRI, $66 \%$ of which were UBOs (unidentified bright objects). 13(22\%) optic pathway gliomas, 7 (11.8\%) non-glioma CNS tumours, $8(13.5 \%)$ had other CNS abnormalities. 10/59 (17\%) required surgical intervention. In 5/ $59(8 \%)$ children the scan was normal. Patients with learning difficulties and neuro-developmental disorders (such as ASD/ADD) had a significantly higher incidence of UBO (90\% vs. 40\%; p < 0.001). There was a higher incidence of non-UBO CNS lesions amongst the group with learning difficulties. 2 asymptomatic patients had incidental findings on scans done for other reasons; 1 of them required surgical intervention.

32/100 children had MRI spine, common indications being scoliosis (40\%) and back/neck pain (28\%). 34\% of these scans were abnormal. Chiari malformation, cervical syrinx and dural ectasia were the most common abnormalities. 5/27 (18\%) needed scoliosis surgery.

Conclusion Targeted imaging in our cohort identified a significant proportion of abnormalities. Children with NF1 and associated neurodevelopmental conditions (learning difficulties, ASD, ADHD) were more likely to have an abnormality on the scans, thus warranting a lower threshold for neuroimaging. For the efficient use of resources, a high index of clinical suspicion is essential for early identification of NF1 related complications.

\section{G198(P) CHILD AND ADOLESCENT PSYCHIATRY TRAINING DURING NEURODISABILITY GRID: AN EXPERIENCE BY THE TRAINEES IN THE UK}

V Mundada. Paediatric Neuroscience, Great Ormond Street Hospital, London, UK

10.1136/archdischild-2015-308599.192
Aim The neurology and neurodisability grid trainees should have a child and adolescent psychiatry experience equivalent to three months full time during their grid programme. The aim of our survey was to gather information and analyse the child and adolescent psychiatry training experience by the current paediatric neurodisability trainees.

Method This was a questionnaire based survey which was sent out to the existing neurodisability grid trainees in the UK via email. Total 10 questions were asked related to the child and adolescent psychiatry training experience.

Results Out of total 26 trainees to whom the survey was sent out, 16 responded (61.5\%). 60\% were working as ST8. The neurodisability grid programme was of two years for $60 \%$ of the trainees. Most of them (73\%) had already started their neuropsychiatry placement. Only 6\% found easy but 26\% thought that it was very difficult to access the training. $15 \%$ had to have a separate honorary contract with the child and family mental health (CAMHS) department. None was on a separate 3 month contract. In terms of satisfaction, one third seemed dissatisfied for various reasons and only $6 \%$ were very satisfied.

Some of the comments were- "CAMHS team members are reluctant to have the neurodisability trainee in the clinic unless it was a pre-school child with possible developmental problems"; "No learning disability CAMHS consultant in the service"; "CAMHS services were not sure of the competencies needed to be achieved"; "Difficult to get the placement and the service was quite stretched"; "Spent a lot of time chasing sessions"; "CAMHS consultants should be involved while submitting the grid posts to RCPCH"

Conclusion Child and adolescent mental health services (CAMHS) are lacking in the expertise and resources required to provide comprehensive assessments and ongoing management for those with developmental disabilities. Our survey highlights the need for the CAMHS service to engage the neurology and neurodisability grid trainees by understanding the competencies they need to achieve in child and adolescent psychiatry. Equally, the respective CSACs could also work in partnership with the child mental health CSAC to design a smooth and well-structured programme for these trainees.

\section{G199(P) AUDIT OF INFORMATION PROVIDED TO PARENTS OF CHILDREN WITH EPILEPSY}

MG Ghazavi. Paediatrics, South Tyneside NHS Foundation Trust, South Shields, UK

\subsection{6/archdischild-2015-308599.193}

Introduction Epilepsy is common in children. Diagnosis may impact negatively on the family. Witnessing a seizure can be very frightening. A wide range of emotions and reactions from the parents are reported once the diagnosis is made. High quality information for the family provided on time can be very beneficial. Involving the child and family in decision making is associated with good outcome and improve compliance.

Aim There are risks associated with epilepsy. Many professionals pay attention to medical management (medication) once a diagnosis is made. However, needs of the child and family for high quality information is ignored. NICE guideline has highlighted what information needs to be provided to the family and what safety measures to be taken. As with no epilepsy clinic locally, I performed an audit to look into our practice and to check it against the standards set by the NICE and National service 